\title{
Symmetry transform in Faddeev-Jackiw quantization of dual models
}

\author{
C. P. Natividade \\ Instituto de Física, Universidade Federal Fluminense, Avenida Litorânea s/n, Boa Viagem, Niterói, 24210-340 Rio de Janeiro, Brazil \\ and Departamento de Física e Química, Universidade Estadual Paulista, Avenida Ariberto Pereira da Cunha 333, \\ Guaratinguetá, 12500-000 São Paulo, Brazil \\ A. de Souza Dutra \\ Departamento de Física e Química, Universidade Estadual Paulista, Avenida Ariberto Pereira da Cunha 333, \\ Guaratinguetá, 12500-000 São Paulo, Brazil \\ H. Boschi-Filho \\ Instituto de Física, Universidade Federal do Rio de Janeiro, Caixa Postal 68528, Rio de Janeiro, 21945-970 Rio de Janeiro, Brazil
} (Received 20 October 1998; published 23 February 1999)

\begin{abstract}
We study the presence of symmetry transformations in the Faddeev-Jackiw approach for constrained systems. Our analysis is based in the case of a particle submitted to a particular potential which depends on an arbitrary function. The method is implemented in a natural way and symmetry generators are identified. These symmetries permit us to obtain the absent elements of the sympletic matrix which complement the set of Dirac brackets of such a theory. The study developed here is applied in two different dual models. First, we discuss the case of a two-dimensional oscillator interacting with an electromagnetic potential described by a ChernSimons term and second the Schwarz-Sen gauge theory, in order to obtain the complete set of non-null Dirac brackets and the correspondent Maxwell electromagnetic theory limit. [S0556-2821(99)04804-3]
\end{abstract}

PACS number(s): 11.10.Kk, 11.15.Tk

\section{INTRODUCTION}

Dual symmetries play a fundamental role in classical electromagnetic theory as realized since the completion of its equations by Maxwell in the last century. In quantum theory, however, these symmetries were not fully appreciated until the works of Montonen and Olive [1] and more recently Seiberg and Witten [2] in 3+1 dimensions and the study of Chern-Simons (CS) theories [3] in 2+1 dimensions. Since these theories have gauge symmetries, they are naturally constrained.

The study of constrained systems consists of a very interesting subject which has been intensively explored by using different techniques [4], alternatively to the pioneering procedure of Dirac [5]. In that original work, the constraints were classified into two categories which have different physical meanings: first-class constraints related to gauge symmetries and second-class ones which represent a reduction of the degrees of freedom. In addition to its applicability, the Dirac method presents some difficulties when one studies systems presenting only second-class constraints and there one verifies the presence of symmetries despite the gauge fixation. This is what happens with $2 \mathrm{D}$ induced gravity where the $\operatorname{SL}(2, R)$ symmetry was not detected by convential methods but by analyzing the anomaly equation by Polyakov [6,7]. Later on, Barcelos-Neto [8] using the Dirac and sympletic methods reobtained this result and also found a Virasoro hidden symmetry in the Polyakov 2D gravity.

From the canonical point of view, the study of symmetries can be approached with the Faddeev-Jackiw (FJ) sympletic procedure [9]. In this approach, the phase space is reduced in such a way that the Lagrangian depends on the first-order velocities. The advantage of this linearization is that the non- null Dirac brackets are the elements of the sympletic matrix [10-12]. For gauge systems, this matrix is singular and has no inverse unless a gauge-fixing term is included. The FJ method is very simple to use; nevertheless, it does not explicitly give all non-null Dirac brackets in the sympletic matrix. Some of them are obtained only by use of the equations of motion [13]. However, this problem may be circumvented if we consider some symmetry transformations in the fields.

In this work we show how to implement this idea by using first in Sec. II an example in one dimension where a particle is submitted to an arbitrary potential which depends on a function which will represent the constraints of the model. It is possible to verify that the generators of the symmetries are given in terms of the zero modes of the sympletic matrix. Then we implement symmetry transformations on the Lagrangian of the system so that new non-null Dirac brackets emerge from the sympletic matrix. These ideas are especially important to discuss dual theories where Dirac brackets involving gauge fields are expected to appear. However, as we are going to show in two different models these brackets do not come from a canonical implementation of the sympletic method. We then show that introducing convenient symmetry transformations, we can obtain the complete set of Dirac brackets of the corresponding dual models. In Sec. III we apply this method to quantize the problem of a charged oscillator in two space dimensions interacting with an electromagnetic field described by a CS term. A similar system has been investigated before using the Dirac method [14] and it can be understood as an extension of the quantum mechanical model of Dunne, Jackiw, and Trugenberger [15]. Two of the present authors have also investigated this system [13] using the FJ method but in a noncanonical way, in the sense that we have not included a field to play the role of the 
momentum of the CS field. This planar system is also interesting to use to explore the role of the canonical quantization of a particle under the influence of a gauge field. Consequently, it can be interpreted like a laboratory to the dimensional reduction approach in other more complicated models [16]. In Sec. IV, we explore the ideas introduced in Sec. II to quantize, from the canonical point of view, the Schwarz-Sen model [17]. The study of symmetry dualities reveals a conflict between electric-magnetic duality symmetries and Lorentz invariance at the quantum level in the Maxwell theory $[18,19]$. In a very interesting way, Schwarz and Sen proposed a four-dimensional action by using two gauge potentials, such that the duality symmetry is established in a local way. As a consequence, the equivalence between this alternative theory and Maxwell's one is demonstrated. Here, we use the features discussed in Sec. II to obtain this equivalence. For our convenience, we choose the Coulomb gauge in the treatment of both gauge theories discussed in Secs. III and IV, so that a parallel of the sympletic structure between that two different dual models can be easily traced. Conclusions and final comments are presented in Sec. V.

\section{SYMMETRY TRANSFORM IN THE FADDEEV-JACKIW APPROACH}

In order to show how the symmetry transformations are related to the zero modes of the sympletic matrix in the FJ approach we have made use of a simple case, where a particle has been submitted to a potential which depends on a constrained function. For a review of FJ method and applications we refer to Refs. [9-13].

Let us start by considering the following Lagrangian:

$$
L^{(0)}=p_{i} \dot{q}_{i}+V(q, p, \Omega),
$$

where the potential is defined as

$$
V(q, p, \Omega)=\lambda \Omega(q, p)-W(q, p),
$$

such that $\Omega(q, p)$ represent the constraints, $\lambda$ a Lagrange multiplier, and $W(q, p)$ the resultant potential. Following the steps of the sympletic method we must build a sympletic matrix which contains the Dirac brackets. Hence, we begin defining the matrix elements [9-13]

$$
\hat{\rho} \equiv\left(\rho_{i j}\right)=\frac{\partial a_{j}}{\partial \xi_{i}}-\frac{\partial a_{i}}{\partial \xi_{j}}
$$

$\xi_{i} \equiv\left(q_{i}, p_{i}\right)$ being the generalized coordinates and $a_{i}$ the coefficients of the velocities in the first-order Lagrangian $L^{(0)}$. Therefore we have, by inspecting $L^{(0)}$, that $a_{q}^{i} \equiv p^{i}$ and then

$$
\rho_{q p}^{i j}=-\frac{\partial a_{q}^{i}}{\partial p_{j}}+\frac{\partial a_{p}^{j}}{\partial q_{i}}=-\delta_{i j},
$$

since $a_{p}^{j}$ vanishes. Now, defining the vector $\xi_{i}=\left(q_{i}, p_{i}, \lambda\right)$ and calculating the respective coefficients, we obtain the matrix

$$
\hat{\rho}^{(0)}=\left(\begin{array}{ccc}
0 & -\delta_{i j} & 0 \\
\delta_{i j} & 0 & 0 \\
0 & 0 & 0
\end{array}\right),
$$

which is obviously singular, since $\operatorname{det} \hat{\rho}^{(0)}=0$. Then, in this case we cannot identify $\hat{\rho}^{(0)}$ as the sympletic matrix. This feature reveals that the system under consideration is constrained [10-12]. A manner to circumvent this problem is to use the constraints conveniently to change the coefficients $a^{i}(\xi)$ in the first-order Lagrangian (2.1) and consequently obtain a rank-2 tensor which could be identified with the sympletic matrix.

In the present case, we can build up an eigenvalue equation with the matrix $\hat{\rho}^{(0)}$ and eigenvectors $v_{i}^{(0)}$ such that

$$
v_{i}^{(0)} \rho^{(0) i j}=0 .
$$

From the variational principle applied to Lagrangian (2.1) we find the condition over the zero modes,

$$
v_{i}^{(0)} \partial^{i} V^{(0)} \equiv \chi^{(0)}
$$

which generates the constraint $\chi^{(0)}$. If we impose that $\chi^{(0)}$ does not evolve in time, we arrive at

$$
\dot{\chi}^{(0)}=\left(\partial_{i} \chi^{(0)}\right) \dot{q}^{i},
$$

and since $\dot{\chi}^{(0)}$ is linear in $\dot{q}^{i}$, we can incorporate this factor into Lagrangian (2.1). This operation means to redefine the coefficients $a_{i}^{(0)}(\xi)$ in the form

$$
\widetilde{a}_{i}^{(0)}(\xi) \rightarrow a_{i}^{(0)}(\xi)+\lambda \partial_{i} \chi^{(0)},
$$

where $\lambda$ is a Lagrange multiplier. Consequently the matrix $\hat{\rho}^{(0)}$ becomes

$$
(\widetilde{\rho})_{i j}=\frac{\partial \widetilde{a}_{j}}{\partial \xi_{i}}-\frac{\partial \widetilde{a}_{i}}{\partial \xi_{j}} .
$$

After completing this, if $\operatorname{det}(\widetilde{\rho})_{i j}$ is still vanishing, we must repeat the above strategy until we find a nonsingular matrix. As has been pointed out in the Refs. [10-12] for systems which involve gauge fields it may occur that the matrix is singular and the eigenvectors $v_{i}^{(m)}$ do not lead to any new constraints. In this case, in order to obtain an invertible matrix, it is necessary to fix some gauge. Such a case will be discussed in the following sections.

Going back to Eq. (2.5), we can see that Eq. (2.8) is satisfied for the eigenvector $v_{i}^{(0)}=(0,0,1)$. On the other hand, from Eq. (2.7) and the Lagrangian (2.1), we get

$$
\begin{aligned}
\chi^{(0)} & =v_{i}^{(0)} \partial^{i} V^{(0)}=v_{\lambda}^{(0)} \frac{\partial V^{(0)}}{\partial \lambda}=0 \\
& \equiv \Omega(p, q),
\end{aligned}
$$

so that $\Omega(p, q)$ is the primary constraint of the theory. In order to include this constraint into the Lagrangian density we can use a new Lagrange multiplier $\eta$ and take 


$$
\begin{aligned}
L^{(1)} & =\left.L^{(0)}\right|_{\Omega=0}+\dot{\eta} \Omega(q, p) \\
& =p_{i} \dot{q}_{i}+\dot{\eta} \Omega(q, p)-W(q, p) .
\end{aligned}
$$

Hence, the new coefficients which contribute to the matrix are $a_{q}^{i}=p_{i}$ and $a_{\eta}^{i}=\Omega$. Then, the iterated matrix $\hat{\rho}^{(1)}$ reads

$$
\hat{\rho}^{(1)}=\left(\begin{array}{ccc}
0 & -\delta_{i j} & \frac{\partial \Omega^{j}}{\partial q_{i}} \\
\delta_{i j} & 0 & \frac{\partial \Omega^{j}}{\partial p_{i}} \\
-\frac{\partial \Omega^{i}}{\partial q_{j}} & -\frac{\partial \Omega^{i}}{\partial p_{j}} & 0
\end{array}\right),
$$

which means that $\operatorname{det} \hat{\rho}^{(1)} \equiv\left\{\Omega^{i}, \Omega^{j}\right\}_{P B}$. Here, there are two possibilities. The first is when $\operatorname{det} \hat{\rho}^{(1)} \neq 0$ and the matrix $\hat{\rho}^{(1)}$ is invertible. The second one occurs when $\operatorname{det} \hat{\rho}^{(1)}=0$. This case is more interesting, since the eigenvectors

$$
v_{i}^{(1)}=\left(-\frac{\partial \Omega}{\partial p_{i}}, \frac{\partial \Omega}{\partial q_{i}}, 1\right)
$$

can be identified as the generators of infinitesimal transformations. This feature will be quite explored in our analysis.

Going back to the matrix given by Eq. (2.13), we notice the absence of the diagonal elements. This is apparently natural since by definition $\hat{\rho}^{(m)}$ is a rank-2 tensor, and in general this tensor is antisymmetric. However, there are cases where the system contains duality symmetry, as, for example, in the Chern-Simons theories $[3,4]$. In order to incorporate these elements into the iterated matrix $\hat{\rho}^{(1)}$, we can suppose that some kind of symmetry transform can be obtained from the zero modes $v_{i}^{(m)}$.

Therefore, let us consider the following transformation in the auxiliary coordinate: ${ }^{1}$

$$
p_{i} \rightarrow p_{i}+f_{i} \Rightarrow \delta p_{i}=\frac{\partial f_{i}}{\partial q_{j}} \delta q_{j}
$$

where $f_{i}=f_{i}(q)$. Consequently, the modified Lagrangian $\widetilde{L}^{(0)}$ is given by

$$
\begin{aligned}
\widetilde{L}^{(0)} & =\left(p_{i}+f_{i}\right) \dot{q}_{i}+\widetilde{V}\left(q_{i}, p_{i}+f_{i}\right) \\
& =\left(p_{i}+f_{i}\right) \dot{q}_{i}+\lambda \widetilde{\Omega}\left(q_{i}, p_{i}+f_{i}\right)-\widetilde{W}\left(q_{i}, p_{i}+f_{i}\right),
\end{aligned}
$$

and by implementing the sympletic method here we obtain the matrix

\footnotetext{
${ }^{1}$ The transformation given by this equation has been suggested in order to make more simple the development of this section. The final result obtained here can be checked via more general transformations as well.
}

$$
\left(\widetilde{\rho}_{i j}\right)^{(1)}=\left(\begin{array}{ccc}
f_{i j} & \delta_{i j} & \frac{\partial \Omega^{j}}{\partial q_{i}} \\
-\delta_{i j} & 0 & \frac{\partial \Omega^{j}}{\partial p_{i}} \\
-\frac{\partial \Omega^{i}}{\partial q_{j}} & -\frac{\partial \Omega^{i}}{\partial p_{j}} & 0
\end{array}\right)
$$

in such a way that, according to Eqs. (2.6) - (2.9), we arrive at

$$
\operatorname{det}\left(\widetilde{\rho}_{i j}\right)^{(1)}=\left\{\widetilde{\Omega}^{i}, \widetilde{\Omega}^{j}\right\},
$$

where $f_{i j} \equiv \partial f_{j} / \partial q_{i}-\partial f_{i} / \partial q_{j}$. Now, since $f_{j}$ is infinitesimal, we can write

$$
\widetilde{\Omega}^{i}\left(q_{j}, p_{j}+f_{j}\right)=\widetilde{\Omega}^{i}\left(q_{j}, p_{j}\right)+\left(\frac{\partial \widetilde{\Omega}^{i}}{\partial p_{k}}\right) \underset{f=0}{f^{k}}
$$

which implies that

$$
\begin{aligned}
\left\{\widetilde{\Omega}^{i}, \widetilde{\Omega}^{j}\right\}_{P B} & =\left\{\Omega^{i}, \Omega^{j}\right\}\left[1+\left(\frac{\partial \widetilde{\Omega}^{i}}{\partial p_{k}}\right)_{f=0} f^{k}\right] \\
& \equiv 0
\end{aligned}
$$

since $\left\{\Omega^{i}, \Omega^{j}\right\}=0$ has been considered here. The above result reveals that the constraint algebra is preserved in front of transformations (2.15). Consequently, the matrix $\left(\widetilde{\rho}^{(1)}\right)_{i j}$ remains singular and the zero modes in this case become

$$
v_{i}^{(1)}=\left(-\frac{\partial \widetilde{\Omega}^{i}}{\partial p_{j}}, \frac{\partial \widetilde{\Omega}^{i}}{\partial q_{j}}-\frac{\partial \widetilde{\Omega}^{i}}{\partial p_{k}} f^{k}, 1\right),
$$

which implies that

$$
v_{i}^{(1)} \widetilde{\rho}_{i j}^{(1)}=\left(0,0,\left\{\widetilde{\Omega}^{i}, \widetilde{\Omega}^{j}\right\}\right),
$$

giving a null vector by virtue of Eq. (2.20). On the other hand, the action of the zero modes on the equations of motion yields

$$
v_{i}^{(1)}\left[\frac{\partial L^{(1)}}{\partial \xi_{i}}-\frac{d}{d t}\left(\frac{\partial L^{(1)}}{\partial \dot{\xi}_{i}}\right)\right]=0=\dot{\eta}\left\{\widetilde{\Omega}^{i}, \widetilde{\Omega}^{j}\right\}-\left\{\widetilde{\Omega}^{i}, \widetilde{W}^{j}\right\},
$$

as a consequence of Eqs. (2.12) and (2.21). This means that no new constraints can arise from the equations of motion. From the above equation we can get

$$
\left\{\widetilde{\Omega}^{i}, \widetilde{W}^{j}\right\}=v_{i}^{(1)} \partial^{i} \widetilde{W},
$$

and by virtue of Eq. (2.20) we conclude that the zero modes are orthogonal to the gradient of the potential $\widetilde{W}$, indicating that they are generators of local displacements on the isopotential surface. Consequently, they generate the infinitesimal transformations; i.e., for some quantity $A(\xi)$ we must have 


$$
\delta A^{\alpha}=\left(\frac{\partial A^{\alpha}}{\partial \xi_{i}} v_{i}\right) \varepsilon
$$

$\varepsilon$ being an infinitesimal parameter. From Eqs. (2.21) and (2.25) we have

$$
\begin{gathered}
\delta q_{i}=-\frac{\partial \widetilde{\Omega}^{i}}{\partial p_{l}} \varepsilon_{l}, \\
\delta p_{i}=\left(\frac{\partial \widetilde{\Omega}^{i}}{\partial q_{l}}-\frac{\partial \widetilde{\Omega}^{i}}{\partial p_{l}} f^{i l}\right) \varepsilon_{l}, \\
\delta \eta=\varepsilon,
\end{gathered}
$$

which permit us to show that the Lagrangian $\widetilde{L}^{(1)}$ becomes

$$
\begin{aligned}
\widetilde{L}^{(1)} & =L^{(1)}+\delta L^{(1)} \\
& =L^{(1)}+\frac{d}{d t}\left(p_{i} \delta q_{i}+\delta \eta \widetilde{\Omega}^{i}\right) \varepsilon,
\end{aligned}
$$

which does not change the original equation of motion. Therefore, the introduction of symmetry transforms into the original Lagrangian $L^{(0)}$ leads to some elements of the sympletic matrix, which are the Dirac brackets. Notice that this result has been obtained without loss of the formal structure of the constraint algebra and the equations of motion. In the following sections we present explicitly examples in field theory where these ideas will be explored in some depth.

\section{OSCILLATOR INTERACTING WITH A CHERN-SIMONS TERM}

Let us now consider the problem of charged particles subjected to a harmonic oscillator potential moving in two dimensions and interacting with an electromagnetic field described by a Chern-Simons term. This problem was inspired by the Dunne-Jackiw-Trugenberger model [15] and has been considered before $[14,13]$ in different situations. Here we want to apply the canonical form of the sympletic method which will lead us to the Dirac brackets but some of them will be missing as we discussed in the previous section. Then we use a convenient transformation to get the complete brackets set. So we start with the Lagrangian ${ }^{2}$

\footnotetext{
${ }^{2}$ Our conventions here are $\epsilon_{012}=\epsilon^{012}=1$ and $g^{\mu \nu}=\operatorname{diag}(-++)$.
}

$$
\begin{aligned}
L= & \frac{m}{2}\left[\dot{q}_{i}(t) \dot{q}^{i}(t)-\omega^{2} q_{i}(t) q^{i}(t)\right] \\
& -e \int d^{2} x A_{0}(t, \vec{x}) \delta(\vec{x}-\vec{q})+e \int d^{2} x A_{i}(t, \vec{x}) \\
& \times \delta(\vec{x}-\vec{q}) \dot{q}^{i}(t)+\theta \int d^{2} x \epsilon_{\mu \nu \rho} A^{\mu}(t, \vec{x}) \partial^{\nu} A^{\rho}(t, \vec{x}),
\end{aligned}
$$

where $q_{i}(t)$ is the particle coordinate with charge $-e$ on the plane $(i=1,2), A_{\mu}(t, \vec{x})$ is the electromagnetic potential $(\mu$ $=0,1,2)$, and $\theta$ is the Chern-Simons parameter. In order to implement the sympletic method here we introduce the auxiliary coordinate $p_{i}(t)$ through the transformation $\dot{q}^{2}$ $\rightarrow 2 p \cdot q-p^{2}[10]$, and define an auxiliary field $\Pi_{i}(t, \vec{x})$ $=\epsilon_{i j} A^{j}(t, \vec{x})$, so that we can write the above Lagrangian as

$$
\begin{aligned}
L^{(0)}= & {\left[m p_{i}(t)-e A_{i}(t, \vec{q})\right] \dot{q}^{i}(t) } \\
& -\theta \int d^{2} x \Pi_{i}(t, \vec{x}) \dot{A}^{i}(t, \vec{x})-V^{(0)},
\end{aligned}
$$

where $A_{i}(t, \vec{q})=\int d^{2} x A_{i}(t, \vec{x}) \delta(\vec{x}-\vec{q})$, and the potential is given by

$$
\begin{aligned}
V^{(0)}= & \frac{m}{2}\left[p_{i}(t) p^{i}(t)+\omega^{2} q_{i}(t) q^{i}(t)\right] \\
& +e A_{0}(t, \vec{q})+2 \theta \int d^{2} x \partial^{i} \Pi_{i}(t, \vec{x}) A_{0}(t, \vec{x})
\end{aligned}
$$

Since this Lagrangian is linear on the velocities, we can identify the sympletic coefficients

$$
\begin{gathered}
a_{q_{i}(t)}^{(0)}=m p_{i}(t)-e A_{i}(t, \vec{q}), \\
a_{A_{i}(t, \vec{x})}^{(0)}=-\theta \Pi_{i}(t, \vec{x}),
\end{gathered}
$$

while the others are vanishing, which lead us to the matrix elements

$$
\begin{gathered}
\rho_{q_{i} p_{j}}^{(0)}=-m \delta_{i j}, \\
\rho_{q_{i} A_{j}}^{(0)}=e \delta_{i j} \delta(\vec{y}-\vec{q}), \\
\rho_{A_{i} A_{j}}^{(0)}=0, \\
\rho_{A_{i} \Pi_{j}}^{(0)}=\theta \delta_{i j} \delta(\vec{x}-\vec{y}) .
\end{gathered}
$$

Defining the sympletic vector to be given by $y^{\alpha}$ $=\left(\vec{q}, \vec{p}, \vec{A}, \vec{\Pi}, A_{0}\right)$ we have the matrix 


$$
\rho_{\alpha \beta}^{(0)}=\left(\begin{array}{ccccc}
0 & -m \delta_{i j} & e \delta_{i j} \delta(\vec{y}-\vec{q}) & 0 & 0 \\
m \delta_{i j} & 0 & 0 & 0 & 0 \\
-e \delta_{i j} \delta(\vec{y}-\vec{q}) & 0 & 0 & \theta \delta_{i j} \delta(\vec{x}-\vec{y}) & 0 \\
0 & 0 & -\theta \delta_{i j} \delta(\vec{x}-\vec{y}) & 0 & 0 \\
0 & 0 & 0 & 0 & 0
\end{array}\right)
$$

which is obviously singular. The zero modes come from the equation

$$
\frac{\partial V^{(0)}}{\partial A_{0}(t, \vec{x})}=e \delta(\vec{x}-\vec{q})+2 \theta \partial^{i} \Pi_{i},
$$

which implies the primary constraint $\chi^{(0)}=e \delta(\vec{x}-\vec{q})+2 \theta \partial^{i} \Pi_{i}$ (Gauss law). Using this constraint we can build up the Lagrangian

$$
L^{(1)}=L^{(0)}+\dot{\lambda} \chi^{(0)},
$$

where $\lambda$ is a Lagrange multiplier, and now the potential reads

$$
V^{(1)}=\left.V^{(0)}\right|_{\chi^{(0)}=0}=\frac{m}{2}\left[p_{i}(t) p^{i}(t)+\omega^{2} q_{i}(t) q^{i}(t)\right] .
$$

The new non-null velocity coefficient is given by $a_{\lambda}^{(1)}=\chi^{(0)}=e \delta(\vec{x}-\vec{q})+2 \theta \partial^{i} \Pi_{i}$, so that we have new matrix elements $\rho_{A_{i} \lambda}^{(1)}=0$ and $\rho_{\Pi_{j} \lambda}^{(1)}=2 \theta \partial_{j} \delta(\vec{x}-\vec{y})$, which lead us to the matrix $\left[y^{\alpha}=(\vec{q}, \vec{p}, \vec{A}, \vec{\Pi}, \lambda)\right]$

$$
\rho_{\alpha \beta}^{(1)}=\left(\begin{array}{ccccc}
0 & -m \delta_{i j} & e \delta_{i j} \delta(\vec{y}-\vec{q}) & 0 & 0 \\
m \delta_{i j} & 0 & 0 & 0 & 0 \\
-e \delta_{i j} \delta(\vec{y}-\vec{q}) & 0 & 0 & \theta \delta_{i j} \delta(\vec{x}-\vec{y}) & 0 \\
0 & 0 & -\theta \delta_{i j} \delta(\vec{x}-\vec{y}) & 0 & 2 \theta \partial_{i} \delta(\vec{x}-\vec{y}) \\
0 & 0 & 0 & -2 \theta \partial_{j} \delta(\vec{x}-\vec{y}) & 0
\end{array}\right),
$$

which is still singular. The zero modes will not lead to any new constraints, and so we have to choose a gauge, which will be the Coulomb one $(\vec{\nabla} \cdot \vec{A}=0)$, and we include it in the Lagrangian via another Lagrange multiplier $\eta$ :

$$
\begin{aligned}
L^{(2)}= & L^{(1)}+\dot{\eta} \vec{\nabla} \cdot \vec{A} \\
= & L^{(0)}+\dot{\lambda} \chi^{(0)}+\dot{\eta} \vec{\nabla} \cdot \vec{A}+V^{(1)} \\
= & {\left[m p_{i}(t)-e A_{i}(t, \vec{q})\right] \dot{q}^{i}(t)-\theta \int d^{2} x \Pi_{i}(t, \vec{x}) \dot{A}^{i}(t, \vec{x}) } \\
& +\dot{\lambda}\left[e \delta(\vec{x}-\vec{q})+2 \theta \partial^{i} \Pi_{i}\right]+\dot{\eta} \vec{\nabla} \cdot \vec{A}+\frac{m}{2}\left[p_{i}(t) p^{i}(t)+\omega^{2} q_{i}(t) q^{i}(t)\right],
\end{aligned}
$$

which implies the additional coefficient $a_{\eta}^{(2)}=\vec{\nabla} \cdot \vec{A}$, and the new element $\rho_{A_{i} \eta}^{(2)}=\partial^{i} \delta(\vec{x}-\vec{y})$. The sympletic tensor can then be identified with the matrix, $y^{\alpha}=(\vec{q}, \vec{p}, \vec{A}, \vec{\Pi}, \lambda, \eta)$,

$$
\rho_{\alpha \beta}^{(2)}=\left(\begin{array}{cccccc}
0 & -m \delta_{i j} & e \delta_{i j} \delta(\vec{y}-\vec{q}) & 0 & 0 & 0 \\
m \delta_{i j} & 0 & 0 & 0 & 0 & 0 \\
-e \delta_{i j} \delta(\vec{y}-\vec{q}) & 0 & 0 & \theta \delta_{i j} \delta(\vec{x}-\vec{y}) & 0 & \partial_{i} \delta(\vec{x}-\vec{y}) \\
0 & 0 & -\theta \delta_{i j} \delta(\vec{x}-\vec{y}) & 0 & 2 \theta \partial_{i} \delta(\vec{x}-\vec{y}) & 0 \\
0 & 0 & 0 & -2 \theta \partial_{j} \delta(\vec{x}-\vec{y}) & 0 & 0 \\
0 & 0 & -\partial_{j} \delta(\vec{x}-\vec{y}) & 0 & 0 & 0
\end{array}\right),
$$

which is not singular and can be inverted to give 


$$
\left.\left(\rho^{(2)}\right)^{\alpha \beta}=\mid \begin{array}{cccccc}
0 & \frac{1}{m} \delta_{i j} & 0 & 0 & 0 & 0 \\
-\frac{1}{m} \delta_{i j} & 0 & 0 & -\frac{e}{m \theta} D_{i j} \delta(\vec{x}-\vec{q}) & 0 & -\frac{e}{m} \frac{\partial^{i}}{\nabla^{2}} \delta(\vec{x}-\vec{q}) \\
0 & 0 & 0 & -\frac{1}{\theta} D_{i j} \delta(\vec{x}-\vec{y}) & 0 & -\frac{\partial^{i}}{\nabla^{2}} \delta(\vec{x}-\vec{y}) \\
0 & \frac{e}{m \theta} D_{i j} \delta(\vec{x}-\vec{q}) & \frac{1}{\theta} D_{i j} \delta(\vec{x}-\vec{y}) & 0 & -\frac{1}{2 \theta} \frac{\partial^{i}}{\nabla^{2}} \delta(\vec{x}-\vec{y}) & 0 \\
0 & 0 & 0 & \frac{1}{2 \theta} \frac{\partial^{i}}{\nabla^{2}} \delta(\vec{x}-\vec{y}) & 0 & -\frac{1}{2} \frac{1}{\nabla^{2}} \delta(\vec{x}-\vec{y}) \\
0 & \frac{e}{m} \frac{\partial^{j}}{\nabla^{2}} \delta(\vec{x}-\vec{q}) & \frac{\partial^{j}}{\nabla^{2}} \delta(\vec{x}-\vec{y}) & 0 & \frac{1}{2} \frac{1}{\nabla^{2}} \delta(\vec{x}-\vec{y}) & 0
\end{array}\right),
$$

where $D_{i j}=\delta_{i j}-\partial_{i} \partial_{j} / \nabla^{2}$. From this result we can write down the following Dirac brackets of the theory:

$$
\begin{gathered}
\left\{q_{i}, p_{j}\right\}=\frac{1}{m} \delta_{i j}, \\
\left\{p_{i}, \Pi_{j}\right\}=-\frac{e}{m \theta} D_{i j} \delta(\vec{x}-\vec{q}), \\
\left\{p_{i}, \eta\right\}=-\frac{e}{m} \frac{\partial^{i}}{\nabla^{2}} \delta(\vec{x}-\vec{q}), \\
\left\{A_{i}, \Pi_{j}\right\}=-\frac{1}{\theta} D_{i j} \delta(\vec{x}-\vec{y}), \\
\left\{A_{i}, \eta\right\}=-\frac{\partial^{i}}{\nabla^{2}} \delta(\vec{x}-\vec{y}), \\
\left\{\Pi_{i}, \lambda\right\}=-\frac{1}{2 \theta} \frac{\partial^{i}}{\nabla^{2}} \delta(\vec{x}-\vec{y}), \\
\{\lambda, \eta\}=-\frac{1}{2} \frac{1}{\nabla^{2}} \delta(\vec{x}-\vec{y}) .
\end{gathered}
$$

However, as was anticipated in Sec. II, some non-null brackets are missing. To overcome this situation let us consider the transformation

$$
\Pi_{i}(t, \vec{x}) \rightarrow \Pi_{i}^{\prime}(t, \vec{x})-\epsilon_{i j} A^{j}(t, \vec{x})
$$

on the Lagrangian of the system. So following the same steps as shown above we find a Lagrangian $L^{(2)^{\prime}}$ which is identical to $L^{(2)}$ except for the substitutions

$$
\begin{aligned}
-\theta \int d^{2} x \Pi_{i}(t, \vec{x}) \dot{A}^{i}(t, \vec{x}) \rightarrow & -\theta \int d^{2} x \Pi_{i}^{\prime}(t, \vec{x}) \dot{A}^{i}(t, \vec{x}) \\
& +\theta \int d^{2} x \epsilon_{i j} A^{j}(t, \vec{x}) \dot{A}^{i}(t, \vec{x})
\end{aligned}
$$

and

$$
2 \theta \dot{\lambda} \partial^{i} \Pi_{i}(t, \vec{x}) \rightarrow 2 \theta \dot{\lambda} \partial^{i} \Pi_{i}^{\prime}(t, \vec{x})-2 \theta \dot{\lambda} \epsilon_{i j} \partial^{i} A^{j}(t, \vec{x}),
$$

so that we find the coefficients

$$
\begin{gathered}
a^{(2)^{\prime}}{ }_{A^{i}(t, \vec{x})}=-\theta \Pi_{i}^{\prime}(t, \vec{x})+\theta \epsilon_{i j} A^{j}(t, \vec{x}), \\
a_{\lambda}^{(2)^{\prime}}=e \delta(\vec{x}-\vec{q})+2 \theta \partial^{i} \Pi_{i}^{\prime}(t, \vec{x})-2 \theta \epsilon_{i j} \partial^{i} A^{j}(t, \vec{x})
\end{gathered}
$$

and the matrix elements

$$
\begin{gathered}
\rho^{(2)^{\prime}}{ }_{A_{i}(t, \vec{x}) A_{j}(t, \vec{y})}=-2 \theta \epsilon_{i j} \delta(\vec{x}-\vec{y}), \\
\rho^{(2)^{\prime}}{ }_{A_{i}(t, \vec{x}) \lambda(t, \vec{y})}=2 \theta \epsilon_{i j} \partial^{j} \delta(\vec{x}-\vec{y}) .
\end{gathered}
$$

These new elements imply another sympletic tensor which can also be inverted. From this inverse we can reobtain the above Dirac brackets and also others which were missing before in our treatment,

$$
\begin{gathered}
\left\{A_{i}, A_{j}\right\}=-\frac{1}{2 \theta} \epsilon_{i j} \delta(\vec{x}-\vec{y}), \\
\left\{A_{i}, \lambda\right\}=\frac{1}{2 \theta} \epsilon_{i j} \partial^{j} \nabla^{-2} \delta(\vec{x}-\vec{y}) .
\end{gathered}
$$

At this point we can identify $\dot{\lambda}$ with $A_{0}$ so that we have the quantized theory expressed in terms of the usual fields. 


\section{SCHWARZ-SEN DUAL MODEL}

Let us begin by introducing the basic idea of the SchwarzSen dual model [17]. The proposal is to treat the problem of the conflict between electric-magnetic duality and manifest Lorentz invariance of the Maxwell theory. We mention, for instance, that by using the Hamiltonian formalism it can be shown that a nonlocal action emerges when one imposes the manifest Lorentz invariance and tries to implement the duality symmetry [18]. In order to circumvent this difficulty Schwarz and Sen proposed the introduction of one more gauge potential into the theory.

In this sense, the model is described by an action that contains two gauge potentials $A_{\mu}^{a}(1 \leqslant a \leqslant 2$ and $0 \leqslant \mu \leqslant 3)$ and is given $b^{3}$

$$
S=-\frac{1}{2} \int d^{4} x\left(B^{a, i} \epsilon^{a b} E^{b, i}+B^{a, i} B^{a, i}\right),
$$

where $E^{a, i}=-F^{a, 0 i}$, while $B^{a, i}=-(1 / 2) \epsilon^{i j k} F_{j k}^{a}$, and $F_{\mu \nu}^{a}$ $=\partial_{\mu} A_{\nu}^{a}-\partial_{\nu} A_{\mu}^{a}$. This action is separately invariant under local gauge transformations

$$
\delta A^{a, 0}=\psi^{a} ; \quad \epsilon A^{a, i}=-\partial^{i} \Lambda^{a}
$$

and duality transformations

$$
A^{a, \mu} \rightarrow \epsilon_{a b} A^{b, \mu}
$$

In terms of the gauge potentials, the corresponding Lagrangian density is given by

$$
\begin{aligned}
\mathcal{L}= & \frac{1}{2} \epsilon^{i j k}\left(\partial_{j} A_{k}^{a}\right) \epsilon_{a b}\left(\dot{A}_{i}^{b}\right) \\
& -\frac{1}{2} \epsilon^{i j k}\left(\partial_{j} A_{k}^{a}\right) \epsilon_{a b}\left(\partial_{i} A_{0}^{b}\right)-\frac{1}{4} F^{a, j k} F_{j k}^{a} .
\end{aligned}
$$

Now, the above Lagrangian density is of first order in time derivative. In order to implement the sympletic method we can define an auxiliary field to make more simple the subsequent calculations. Hence, let us consider the field

$$
\begin{aligned}
\Pi^{a, i} & =\epsilon_{a b} \epsilon^{i j k}\left(\partial_{j} A_{k}^{b}\right) \\
& \equiv \vec{\Pi}^{a}=\epsilon_{a b} \nabla \times \vec{A}^{b},
\end{aligned}
$$

and the Lagrangian density (4.4) becomes

$$
\begin{aligned}
\mathcal{L}^{(0)} & =\frac{1}{2} \vec{\Pi}^{a} \cdot \dot{\vec{A}}^{a}-\frac{1}{2} \vec{\Pi}^{a} \cdot \nabla A_{0}^{a}-\frac{1}{2} \vec{\Pi}^{a} \cdot \vec{\Pi}^{a} \\
& \equiv \frac{1}{2} \vec{\Pi}^{a} \cdot \dot{\vec{A}}^{a}-V^{(0)},
\end{aligned}
$$

where $V^{(0)}=\frac{1}{2} \vec{\Pi}^{a} \cdot \nabla A_{0}^{a}+\left(\frac{1}{2}\right) \vec{\Pi}^{a} \cdot \vec{\Pi}^{a}$. Therefore, the sympletic vector will be given as $\vec{\xi}^{(0)}=\left(\vec{A}^{a}, \vec{\Pi}^{a}, A_{0}^{a}\right)$. From the

\footnotetext{
${ }^{3}$ Our conventions are $\epsilon^{12}=1=-\epsilon^{21}$ and $1 \leqslant i, j, k \leqslant 3$.
}

developments of Sec. II, it is easy to verify that the sympletic matrix correspondent to $\mathcal{L}^{(0)}$ is singular. The zero-mode vector in this case will be $v^{(0)}=\left(0,0, v_{A_{0}}^{(0)}\right)$ and the use of Eqs. (2.6)-(2.8) will give origin to the constraint

$$
\chi=\nabla \cdot \vec{\Pi}^{a}=0,
$$

which can be incorporated into the new Lagrangian density via a Lagrange multiplier. Consequently, we have

$$
\begin{aligned}
\mathcal{L}^{(1)} & =\left.\mathcal{L}^{(0)}\right|_{\chi=0}+\dot{\lambda} \nabla \cdot \vec{\Pi}^{a} \\
& =\frac{1}{2} \vec{\Pi}^{a} \cdot \dot{\vec{A}}^{a}+\dot{\lambda} \nabla \cdot \vec{\Pi}^{a}-\frac{1}{2} \vec{\Pi}^{a} \cdot \vec{\Pi}^{a},
\end{aligned}
$$

which leads to another singular matrix

$$
\left(\rho_{a b}^{(1)}\right)_{i j}=\left(\begin{array}{ccc}
0 & -\delta_{i j} & 0 \\
\delta_{i j} & 0 & -\partial_{i}^{x} \\
0 & \partial_{j}^{x} & 0
\end{array}\right) \delta_{a b} \delta(\vec{x}-\vec{y}),
$$

where the sympletic vector has components $\xi^{(1)}$ $=\left(\vec{A}^{a}, \vec{\Pi}^{a}, \lambda\right)$. On the other hand, the use of Eq. (2.7) implies that

$$
\vec{v}_{A^{a}}-\nabla v_{\lambda}=0
$$

and no new constraints are generated. Here, we remark that the above relation can be used to derive the well-known gauge symmetry

$$
\delta \vec{A}^{a}=\nabla \lambda, \quad \delta \vec{\Pi}^{a}=0, \quad \delta A_{0}^{a}=\dot{\lambda} .
$$

Hence, in this step it is necessary to impose a gauge fixing. If we adopt the Coulomb gauge, as was done in the previous section, the new Lagrangian density becomes

$$
\begin{aligned}
\mathcal{L}^{(2)} & =\left.\mathcal{L}^{(1)}\right|_{\nabla \cdot \vec{A}^{a}=0}+\dot{\eta}\left(\nabla \cdot \vec{A}^{a}\right) \\
& =\frac{1}{2} \vec{\Pi}^{a} \cdot \dot{\vec{A}}^{a}+\dot{\lambda}\left(\nabla \cdot \vec{\Pi}^{a}\right)+\dot{\eta}\left(\nabla \cdot \vec{A}^{a}\right)-\frac{1}{2} \vec{\Pi}^{a} \cdot \vec{\Pi}^{a},
\end{aligned}
$$

and the sympletic matrix, given by

$$
\left(\rho_{a b}^{(2)}\right)_{i j}=\left(\begin{array}{cccc}
0 & -\delta_{i j} & 0 & -\partial_{i}^{x} \\
\delta_{i j} & 0 & -\partial_{i}^{x} & 0 \\
0 & \partial_{j}^{x} & 0 & 0 \\
\partial_{j}^{x} & 0 & 0 & 0
\end{array}\right) \delta_{a b} \delta(\vec{x}-\vec{y}),
$$

can be inverted to give

$$
\begin{aligned}
\left(\rho_{a b}^{(2)}\right)_{i j}^{-1}= & \left(\begin{array}{cccc}
0 & -\delta_{a b} D_{i j} & 0 & \partial_{i}^{x} \nabla^{-2} \\
\delta_{a b} D_{i j} & 0 & \partial_{i}^{x} \nabla^{-2} & 0 \\
0 & -\partial_{j}^{x} \nabla^{-2} & 0 & \nabla^{-2} \\
-\partial_{j}^{x} \nabla^{-2} & 0 & -\nabla^{-2} & 0
\end{array}\right) \\
& \times \delta(\vec{x}-\vec{y}),
\end{aligned}
$$


where $D_{i j}=\delta_{i j}+\partial_{i}^{x} \partial_{j}^{x} \nabla^{-2}$. The sympletic vector now is $\xi^{(2)}=\left(\vec{A}^{a}, \vec{\Pi}^{a}, \lambda, \eta\right)$; therefore, from the above matrix we get

$$
\left\{\vec{A}^{a}(\vec{x}), \vec{\Pi}^{b}(\vec{y})\right\}_{D}=-\delta_{a b}\left(\delta_{i j}+\frac{\partial_{i}^{x} \partial_{j}^{x}}{\nabla^{2}}\right) \delta(\vec{x}-\vec{y}),
$$

which agrees with the result in Ref. [19] obtained from the Dirac procedure. It is important to notice that the matrix (4.14) presents only one bracket, since by virtue of the dual symmetry it must contain diagonal elements like $\left\{\vec{A}^{a}(\vec{x}), \vec{A}^{b}(\vec{y})\right\}_{D}$. This feature can be interpreted by considering the bracket (4.15) as a dynamical one. The part of the sympletic matrix (4.14) related to symmetries cannot be identified directly. However, this term can be generated by means of a convenient symmetry transformation.

In order to implement this, let us consider the following transformation into the Lagrangian density (4.6):

$$
\vec{\Pi}^{a} \rightarrow \vec{\Pi}^{a \prime}-\epsilon_{a b} \nabla \times \vec{A}^{b},
$$

so that we rewrite it as

$$
\begin{aligned}
\mathcal{L}^{(0)^{\prime}}= & +\frac{1}{2}\left(\vec{\Pi}^{a \prime}-\epsilon_{a b} \nabla \times \vec{A}^{b}\right) \cdot\left(\dot{\vec{A}}^{a}-\nabla A_{0}^{a}\right) \\
& -\frac{1}{2}\left(\vec{\Pi}^{a \prime}-\epsilon_{a b} \nabla \times \vec{A}^{b}\right)^{2} .
\end{aligned}
$$

Now, by using Eqs. (2.6) and (2.7), it is easy to verify the presence of the constraint

$$
\chi^{\prime}=\nabla \cdot \vec{\Pi}^{a \prime}
$$

and, consequently,

$$
\mathcal{L}^{(1)^{\prime}}=\left.\mathcal{L}^{(0)^{\prime}}\right|_{\chi^{\prime}=0}+\dot{\alpha}\left(\nabla \cdot \vec{\Pi}^{a \prime}\right) .
$$

The singular matrix corresponding to the above Lagrangian density is given by

$$
\left(G_{a b}^{(1)}\right)_{i j}=\left(\begin{array}{ccc}
\epsilon_{a b} \epsilon_{i j k} \partial^{k} & -\delta_{a b} \delta_{i j} & 0 \\
\delta_{a b} \delta_{i j} & 0 & -\delta_{a b} \partial_{i}^{x} \\
0 & \delta_{a b} \partial_{j}^{x} & 0
\end{array}\right) \delta(\vec{x}-\vec{y}) .
$$

Then, from Eq. (2.7) we obtain the zero modes

$$
\vec{v}_{A^{a}}=\nabla v_{\alpha^{a}}, \quad \vec{v}_{\Pi^{a}}=\nabla \times\left(\epsilon_{a b} \vec{v}_{\vec{A}^{b}}\right),
$$

which confirm the two expected symmetries

$$
\begin{gathered}
\delta \vec{A}^{a}=\nabla \alpha^{a} \quad \text { (gauge) } \\
\delta \vec{\Pi}^{a \prime}=\nabla \times\left(\epsilon_{a b} A^{b}\right) \quad(\text { dual }),
\end{gathered}
$$

and no new constraints are generated. Therefore, we can adopt a gauge fixing. Choosing again the Coulomb gauge we arrive at

$$
\begin{aligned}
\mathcal{L}_{G F}= & \frac{1}{2}\left[\left(\dot{\vec{A}}^{a}-\nabla \dot{\alpha}^{a}-\vec{\Pi}^{a \prime}+\epsilon_{a b} \nabla \times \vec{A}^{b}\right) \cdot \vec{\Pi}^{a \prime}\right. \\
& \left.-\left(\nabla \times \vec{A}^{a}\right)^{2}-\left(\dot{A}^{a}-\nabla \dot{\alpha}^{a}\right) \epsilon_{a b} \nabla \times \vec{A}^{b}\right]_{\nabla \cdot \vec{A}^{a}=0} .
\end{aligned}
$$

Identifying $\dot{\alpha}^{a} \equiv A_{0}^{a}$, we get $\vec{E}^{a}=-\dot{A}^{a}+\nabla A_{0}^{a}$, and consequently the gauge-fixed Lagrangian density becomes

$$
\begin{aligned}
\mathcal{L}_{G F}^{\prime}= & \frac{1}{2}\left(-\vec{E}^{a}-\vec{\Pi}^{a \prime}+\epsilon_{a b} \nabla \times \vec{A}^{b}\right) \cdot \vec{\Pi}^{a \prime} \\
& -\frac{1}{2}\left(\nabla \times \vec{A}^{a}\right)^{2}-\delta \mathcal{L}_{G F}
\end{aligned}
$$

and the gauge-fixing term can be written as

$$
\begin{aligned}
\delta \mathcal{L}_{G F} & =\frac{1}{2}\left(\vec{E}^{a}+\vec{\Pi}^{a \prime}\right) \delta \vec{\Pi}^{a \prime} \\
& =\frac{1}{2}\left(\vec{E}^{a}+\vec{\Pi}^{a \prime}\right) \delta\left(\epsilon_{a b} \nabla \times \vec{A}^{b}\right) \\
& =-\frac{1}{2} \nabla \times\left(\vec{E}^{a}+\vec{\Pi}^{a \prime}\right) \cdot \delta\left(\epsilon_{a b} \vec{A}^{b}\right)+\text { surface terms, }
\end{aligned}
$$

where we used Eq. (4.23).

Before going on, it is important to make some remarks. First of all, we mention that from Eq. (4.25) it is easy to infer that the Dirac bracket between the gauge fields in this case is given by

$$
\left\{\vec{A}^{a}(\vec{x}), \vec{A}^{b}(\vec{y})\right\}_{D}=\epsilon_{a b} \nabla^{-2} \nabla \times \delta(\vec{x}-\vec{y}),
$$

which gives rise to a nonlocal commutation relation for the $\vec{A}^{a}$ field. This relation was obtained here within the context of the sympletic methodology starting from the use of the symmetry transform given by Eq. (4.16).

Another interesting feature is that from the use of Eq. (4.16) and of the gauge-fixed Lagrangian density (4.25) we can show the equivalence between the Schwarz-Sen model and Maxwell theory. From Eq. (4.21), we notice that the variations over $\epsilon_{a b} \vec{A}^{b}$ lead to

$$
\nabla \times\left(\vec{\Pi}^{a \prime}+\vec{E}^{a}\right)=0
$$

and since at this stage the Gauss law can be used, we conclude that

$$
\delta \vec{\Pi}^{a \prime}=-\delta \vec{E}^{a}=\nabla \times\left(\epsilon_{a b} \vec{A}^{b}\right) .
$$

Going back to Eq. (4.30), taking $a=1, b=2$, we have

$$
\mathcal{L}_{G F}^{\prime} \rightarrow \mathcal{L}_{M}=\frac{1}{2}\left(\vec{E}^{1} \cdot \vec{E}^{1}-\vec{B}^{1} \cdot \vec{B}^{1}\right),
$$

which is the Maxwell Lagrangian density. From the Gauss law $\nabla \cdot \vec{\Pi}^{a \prime}=0$ and Eq. (4.28) we find that the vector $\vec{u}^{a}$ $=\vec{\Pi}^{a}+\vec{E} \equiv 0$ implies that $\nabla \cdot \vec{E}^{a}=0$. It is important to notice 
that the $\vec{\Pi}^{a \prime}(\vec{x})$ field is not the canonical momentum of the electromagnetic theory but it represents here an auxiliary field in order to implement the Faddeev-Jackiw method.

\section{COMMENTS AND CONCLUSIONS}

In this work, we study the role of the symmetry transformations in the Faddeev-Jackiw approach. We verify that the generators of such a transformation can be represented in terms of the zero-mode vectors of the singular presympletic matrix. Since the inverse of the sympletic matrix contains elements which define the Dirac brackets of the constrained system, it is natural to ask what happens when some brackets do not appear in this inverse matrix. In our interpretation, these elements are associated with some kind of symmetry transform which, on the other hand, are generated by zeromode vectors. Hence, after a convenient symmetry transformation we can complete the set of the fundamental brackets of the models in question.
Here, we explore this strategy in three different situations: first, for the case where a particle is submitted to a "constrained potential." After we discuss the case of an oscillator in two space dimensions coupled to a Chern-Simons gauge field and finally the Schwarz-Sen dual model for which our main goal was to obtain the corresponding Dirac brackets and how to describe its equivalence with the Maxwell theory. In this point the zero modes played a very important role.

\section{ACKNOWLEDGMENTS}

The authors acknowledge partial financial support from Conselho Nacional de Desenvolvimento Científico e Tecnológico, CNPq, Brazil. A.S.D also acknowledges partial financial support from Fundação de Aruparo à Pesquisa do Estado de São Paulo, FAPESP.
[1] C. Montonen and D. Olive, Phys. Lett. 72B, 117 (1977); see also P. Goddard, J. Nuyts, and D. Olive, Nucl. Phys. B125, 1 (1977).

[2] N. Seiberg, Phys. Lett. B 318, 469 (1993); Phys. Rev. D 49, 6857 (1994); N. Seiberg and E. Witten, Nucl. Phys. B426, 19 (1994); B430, 485(E) (1994).

[3] R. Jackiw and S. Templeton, Phys. Rev. D 23, 2291 (1981); J. Schonfeld, Nucl. Phys. B185, 157 (1981); S. Deser, R. Jackiw, and S. Templeton, Phys. Rev. Lett. 48, 975 (1982); Ann. Phys. (N.Y.) 140, 372 (1982).

[4] M. Henneaux and C. Teitelboim, Quantization of Gauge Systems (Princeton University Press, New York, 1992).

[5] P. A. M. Dirac, Lectures on Quantum Mechanics (Yeshiva University, New York, 1964).

[6] A. M. Polyakov, Mod. Phys. Lett. A 2, 893 (1987).

[7] G. Knizhnik, A. M. Polyakov, and A. B. Zamolodchikov, Mod. Phys. Lett. A 5, 819 (1988).

[8] J. Barcelos-Neto, Phys. Rev. D 49, 1012 (1994).

[9] L. D. Faddeev and R. Jackiw, Phys. Rev. Lett. 60, 1692 (1988).
[10] J. Barcelos-Neto and C. Wotzasek, Mod. Phys. Lett. A 7, 1737 (1992); Int. J. Mod. Phys. A 7, 4981 (1992).

[11] H. Montani, Int. J. Mod. Phys. A 8, 4319 (1993).

[12] C. Wotzasek, Ann. Phys. (N.Y.) 243, 76 (1995).

[13] C. P. Natividade and H. Boschi-Filho, Mod. Phys. Lett. A 11, 69 (1996).

[14] T. Matsuyama, J. Phys. A 23, 5241 (1990).

[15] G. V. Dunne, R. Jackiw, and G. A. Trugenberger, Phys. Rev. D 41, 661 (1990).

[16] R. L. P. G. Amaral and C. P. Natividade, Phys. Rev. D 58, 127701 (1998).

[17] J. H. Schwarz and A. Sen, Nucl. Phys. B411, 35 (1994).

[18] S. Deser and C. Teitelboim, Phys. Rev. D 13, 1592 (1976); I. Martin and A. Restuccia, Phys. Lett. B 323, 311 (1994); D. Sorokin and M. Town, ibid. 352, 59 (1995); P. Pasti, D. Sorokin, and M. Tonin, Phys. Rev. D 52, R4277 (1995).

[19] H. O. Girotti, Phys. Rev. D 55, 5136 (1997); H. O. Girotti, M. Gomes, V. O. Rivelles, and A. J. da Silva, ibid. 56, 6615 (1997). 